\title{
Analyse du discours institutionnel et sociologie compréhensive : vers une anthropologie des discours institutionnels
}

Institutional discourse analysis and comprehensive sociology: the beginning of an anthropology of institutional discourses

Análisis del discurso institucional y sociología comprensiva : hacia una antropología de los discursos institucionales

Claire Oger et Caroline Ollivier-Yaniv

\section{OpenEdition}

Journals

Édition électronique

URL : https://journals.openedition.org/mots/8423

DOI : $10.4000 /$ mots.8423

ISSN : 1960-6001

Éditeur

ENS Éditions

Édition imprimée

Date de publication : 1 mars 2003

Pagination : 125-145

ISBN : 2-84788-027-5

ISSN : 0243-6450

\section{Référence électronique}

Claire Oger et Caroline Ollivier-Yaniv, « Analyse du discours institutionnel et sociologie compréhensive : vers une anthropologie des discours institutionnels », Mots. Les langages du politique [En ligne], 71 | 2003, mis en ligne le 05 mai 2008, consulté le 23 avril 2022. URL : http:// journals.openedition.org/mots/8423; DOI : https://doi.org/10.4000/mots.8423 
Claire OGER ${ }^{\circ}$

Caroline OLLIVIER-YANIV ${ }^{\circ \circ}$

\section{Analyse du discours institutionnel et sociologie compréhensive: vers une anthropologie des discours institutionnels}

Les institutions, loin d'avoir cette existence immédiate, identique pour tous, [...] ont en fait des visages multiples inhérents à la relation que chaque classe d'individus entretient avec elle en fonction de ses enjeux.

Bernard Lacroix, $1992^{1}$

Les réflexions qui suivent constituent la troisième étape d'un travail commun $^{2}$, qui porte sur les relations entre l'institution militaire et les médias et auquel nous avons souhaité donner une dimension réflexive: plutôt qu'à une division du travail entre disciplines, c'est en effet à une confrontation des résultats, mais aussi des méthodes et des concepts que nous tentons de nous livrer. Bien plus, ces relations interdisciplinaires nous semblent pouvoir être formulées sous la forme d'échanges entre analyse du discours institutionnel et sociologie compréhensive ${ }^{3}$, lesquels permettraient de dépasser les simples «importations» entre champs de recherche.

- CREC- Saint-Cyr. CEDITEC-E.A. 3119.

$\circ$ Université Paris-XII, CEDITEC-E.A. 3119.

1. «Le politiste et l'analyse des institutions. Comment parler de la présidence de la République?», 1992, dans B. Lacroix et J. Lagroye (dir.), Le président de la République Usages et genèses d'une institution, Presses de Sciences po.

2. Cet article reprend et développe le texte d'une communication proposée aux journées du GRAM (Groupe d'Analyse des Médias, SFSIC) le 15 mars 2002.

3. Selon les termes de M. Weber, 1995, Économie et société - Les catégories de la sociologie (tome 1) (1956), Paris, Pocket-Agora. 
Cette direction nous a été suggérée par une première étape de ce travail, menée avec Marie-Anne Paveau, au cours de laquelle nous nous étions attachées plus particulièrement aux relations professionnelles entre journalistes et militaires telles qu'elles étaient évoquées par les militaires ${ }^{4}$.

C'est cette problématique qui nous a ensuite conduites à réfléchir sur les résonances que produisaient les rapprochements entre deux de nos corpus: un corpus d'entretiens suscités et recueillis par Caroline OllivierYaniv auprès des anciens chefs du SIRPA ${ }^{5}$, et un corpus de textes institutionnels (qui comportaient des documents internes à l'institution et des articles de la presse militaire) étudié par Claire Oger ${ }^{6}$.

L'examen réflexif de nos résultats produisait en effet un éclairage nouveau: il permettait de rendre compte, au moins en partie, des phénomènes de tension et de contradiction (dénégations, doubles contraintes...) qui caractérisent le discours militaire sur les journalistes. Au risque de simplifier, résumons en disant que les modalités de recueil de l'entretien facilitaient l'évocation de relations professionnelles et personnelles routinisées, voire socialement valorisantes, entre les chefs militaires chargés de la communication de la défense et les quelques journalistes de défense bien connus d'eux. Le discours institutionnel portait plus nettement la marque des contraintes collectives qui pèsent tant sur l'énonciation de prescriptions officielles que sur les échanges entre les militaires (envisagés collectivement comme institution ou comme communauté) et l'ensemble des professionnels des médias (envisagés de manière anonyme au travers de leur fonction et de leurs pratiques).

Ainsi le rapprochement des corpus conduisait à s'interroger sur la manière dont les deux méthodes mobilisées constituaient des «dispositifs d'écoute ${ }^{7}$ différents et complémentaires : les modalités de recueil induisent une distribution différente entre ce qu'on pourrait appeler par approximation

4. Pour un accès à ces travaux, on pourra se reporter à leur publication dans la revue Langage et Société 94, décembre 2000, Paris, MSH: "Les frontières discursives de la militarité» de M.-A. Paveau, «De l'esprit de corps au corps du texte - Cohésion militaire et dissolution journalistique» de C. Oger et "Quels "professionnels" de la communication et des relations avec les médias à la Défense? Carrière militaire et communication» de C. OllivierYaniv.

5. Service d'information et de relations publiques des armées (juillet 1977 -mars 1998).

6. C. Oger et C. Ollivier-Yaniv, 2001, «Approches du discours militaire sur la communication; analyse du discours institutionnel et entretiens ethnographiques », Actes du $12^{e}$ Congrès National des Sciences de l'Information et de la Communication, SFSIC, Paris, p. $157-164$.

7. B. François et E. Neveu, 1999, Espaces publics mosaïques; acteurs, arènes et rhétoriques des débats publics contemporains, Rennes, Presses Universitaires de Rennes, p. 6. 
le discours «individuel» des acteurs et le discours «collectif» de la communauté et/ou de l'institution militaires ${ }^{8}$.

Après avoir présenté nos approches respectives, puis la manière dont elles proposent des inclusions inversées du discursif et du social, nous les aborderons successivement en essayant de montrer quel peut être l'apport de la sociologie à l'analyse du discours et celui de l'analyse du discours à la sociologie.

\section{Deux approches de l'institution en discours}

\section{Les cercles de l'institution}

Le terme d'institution appelle tout d'abord un commentaire: nous considérons que les définitions du terme institution se déploient en cercles concentriques autour d'un cœur constitué par une définition juridique restrictive:

- Nous entendons par «discours institutionnel», au sens strict, le discours produit officiellement par un énonciateur singulier ou collectif qui occupe une position juridiquement inscrite dans l'appareil d'État, qu'il soit fonctionnaire ou représentant politique.

- Au-delà, un deuxième cercle comprend le discours produit par les mêmes énonciateurs en dehors des contextes officiels (exemple: le discours produit par un ministre dans le cadre d'interviews avec des journalistes, par un chef militaire en activité dans un entretien avec un chercheur, par un militaire dans les «libres opinions» du magazine Armées d'aujourd'hui...).

Il est important de distinguer ces deux cercles, car à notre sens, le statut de ces deux discours n'est pas le même: seul le premier a véritablement une valeur performative au sens où il peut comporter des conséquences juridiques, y compris des formes de coercition. La distinction n'est donc nullement de pure forme.

- Un troisième cercle pourrait comprendre le discours produit par des énonciateurs similaires dans des contextes moins contraints (militaires en retraite s'exprimant dans la revue Le Casoar, revue de l'association privée des anciens saint-cyriens, ou récemment dans Libération - général Mer-

8. Cette distinction doit bien sûr être nuancée puisque le discours individuel est pris dans l'institution et que derrière la voix collective et apparemment anonyme de l'institution se trouvent les individus qui occupent les quelques places d'où émane le discours officiel... 
cier - témoignages de hauts fonctionnaires sur leurs fonctions passées comme celui de Simon Nora dans Le débat).

- Enfin, au sens large, le discours institutionnel (institutionnalisé et institutionnalisant) peut comprendre l'ensemble des discours que l'on peut considérer à des degrés divers comme des discours "autorisés» dans un milieu donné, sans référence nécessaire à l'État (productions des syndicats, des états-majors des partis politiques, chartes des associations professionnelles, programmes et règlements d'écoles privées...) ${ }^{9}$.

Par conséquent, institution peut en venir à désigner l'ensemble des contraintes qui pèse sur l'exercice de la fonction énonciative:

Par «institution», on n'entendra pas seulement ces structures exemplaires que sont l'armée ou l'Église, mais plus largement, tout dispositif qui délimite l'exercice de la fonction énonciative, le statut des énonciateurs comme celui des destinataires, les types de contenus que l'on peut et doit dire, les circonstances d'énonciation légitimes pour un tel questionnement (D. Maingueneau ${ }^{10}$.

\section{L'analyse du discours institutionnel}

Une telle conception conduit à solliciter des corpus emboités, correspondant aux divers cercles du discours institutionnel, qui peuvent être confrontés les uns aux autres sans que soit oubliées les discontinuités entre les différentes positions énonciatives que nous venons de définir.

Le travail mené par C. Oger se place dans la perspective d'une analyse du discours (AD) qui mobilise notamment le concept de formation discursive tel qu'il a été reformulé par D. Maingueneau. Il consiste principalement à étudier des textes institutionnels prescriptifs (méthodes, rapports de jurys, règlements, directives...) pour en dégager les traits d'un modèle discursif (et cognitif) ${ }^{11}$ propre à une communauté donnée: il s'agit dans un premier temps de dégager un ensemble de traits cohérents formant une

9. Voir la notion de «groupement social légitimé» chez M. Douglas, et ses corollaires le «collectif de pensée» (moins contraignant) et le «style de pensée» (plus contraignant), empruntés à Fleck. Par ailleurs, Fleck analyse également la structure interne des groupes en termes de centre (élites) et de périphérie (qui reprend les idées de l'élite). M. Douglas, 1999, Comment pensent les institutions, Paris, La Découverte, p. 37.

10. 1991, L'Analyse du Discours; introduction aux lectures de l'archive, Paris, Hachette, p. 18.

11. Les points plus particulièrement étudiés sont ceux qui se trouvent à l'articulation du discursif et du cognitif (notions de "plan» et de «méthode», métaphores privilégiées, reformulations, modalités de l'argumentation par autorité et interdiscours légitime). 
configuration qui définit, pour cette communauté, les conditions d'un exercice légitime de la fonction énonciative ${ }^{12}$. On peut ensuite mettre en relation ce modèle avec les pratiques professionnelles telles qu'elles sont décrites par la communauté étudiée et la manière dont celle-ci s'organise autour d'une mémoire commune.

Cette approche a été adoptée dans le cadre d'une thèse sur les épreuves de culture générale dans trois concours de la fonction publique (École de guerre pour l'Armée de terre, École nationale d'administration et École nationale de la magistrature), ainsi que dans le cadre du travail commun auquel nous nous référons aujourd'hui.

\section{La sociologie compréhensive}

D’un point de vue théorique, les plus consolidées des institutions (le premier cercle évoqué précédemment) gagnent à être envisagées à partir de leur processus de consolidation ${ }^{13}$ et par suite, en prenant en considération les acteurs qui les représentent et les manifestent. Le fait de «remplacer le contenu conventionnel d'un concept par le sens de ce concept en tant que forme d'action collective ${ }^{14}$ nous inscrit ici dans une approche relationnelle des phénomènes institutionnels, laquelle s'oppose à toute forme de substantialisme ${ }^{15}$ ou d'objectivisme qui voit et fait voir l'institution «comme une réalité existant indépendamment de ceux qui l'incarnent, indépendamment en particulier de ce qu'ils sont et de ceux qu'ils font ${ }^{16}$.

Dans cette perspective et du point de vue de la méthode, le choix d'une posture sociologique «compréhensive», selon l'acception de Max Weber,

12. Voir D. Maingueneau qui cite M. Foucault, lorsqu'il évoque l'AD comme une forme d'analyse des énoncés fondée sur leur mise en contexte, en tant que ces énoncés « définissent dans l'espace social une certaine identité énonciative historiquement circonscriptible» (D. Maingueneau, 1991, p. 18).

13. Pour une compréhension des institutions en termes d' «institutionnalisation», voir en particulier B. Lacroix et J. Lagroye (dir.), 1992.

14. H. S. Becker, 2002, 1998, Les ficelles du métier - comment conduire sa recherche en sciences sociales, Paris, La Découverte, p. 232.

15. Ainsi que le propose en particulier N. Elias dans Qu'est-ce que la sociologie? (1970), 1993, Paris, Pocket-Agora, p. 90-91: «La notion de «fonction», comme celle de «puissance», doit être comprise en tant que «concept relationnel» [...] Ceci n'apparait clairement et distinctement que si l'on considère toutes les fonctions, même les fonctions des institutions, comme aspects des relations entre les hommes, que ce soit entre individus ou entre groupes » (C'est nous qui soulignons).

16. B. Lacroix, 1992, p. 43. 
apparait pertinent en tant qu'il s'agit d'une science de l'activité humaine qui considère «l'individu isolé et son activité comme l'unité de base » ${ }^{17}$ et qui se propose de la comprendre par interprétation.

Ce n'est pas seulement la nature particulière du langage, mais aussi celle de notre pensée qui fait que les concepts par lesquels nous saisissons une activité laissent apparaitre celle-ci sous la forme d'une réalité durable, d'une structure choséifiée ou d'une structure "personnifiée», ayant une existence autonome. [...] Des concepts comme ceux d' «État», d' «association», de «féodalité» ou d'autres semblables désignent, d'une manière générale, du point de vue de la sociologie, des catégories représentant des formes déterminées de la coopération humaine; sa tâche consiste à les réduire à une activité «compréhensible», ce qui veut dire sans exception aucune, à l'activité des individus isolés qui y participent (M. Weber, 1913) ${ }^{18}$.

Comprendre par interprétation signifie, pour le chercheur, se donner des modalités d'accès à ce qui reste mystérieux ou inaccessible à son intellect et à ses affects ${ }^{19}$, les «valeurs» d'après lesquelles un acteur social se comporte ne constituant pas le moindre de ces mystères. Les acteurs sociaux sont ici considérés comme dépositaires d'un savoir multiforme qu'il s'agit de saisir de l'intérieur, notamment par le biais du système de valeurs des individus eux-mêmes.

L'articulation entre discours et contexte social, fondatrice de l'AD en tant qu'elle la distingue du strict domaine linguistique, apparait ainsi également structurante de la sociologie compréhensive. On y observe un mouvement en quelque sorte inverse de celui de l'AD: on part du fait social pour prendre en considération son sens endogène, c'est-à-dire l'activité, le vécu et les représentations qui en sont formulées par les acteurs sociaux, particulièrement au moyen de la mise en langage et du discours. Il s'en suit une méthode empirique qui consiste à prendre en considération ce qui est visible en tant qu'accomplissement du sens dirigé vers autrui, et dont la publicité est par définition nécessaire ${ }^{20}$. Autrement dit encore, la

17. M. Weber, 1992 (1913) «Essai sur quelques catégories de la sociologie compréhensive », dans Essais sur la théorie de la science, Paris, Pocket-Agora, p. 318.

18. Ibid., p. 19.

19. On retrouve ici l'idée weberienne selon laquelle il faut chercher à atteindre l'activité réelle en tant qu'elle est «influencée par des irrationalités de toutes sortes (affections, erreurs, valeurs)» (1995, Économie et société, p. 31).

20. Les pratiques et les actions à observer se déploient d'ailleurs dans toutes sortes de dimensions symboliques : linguistiques mais aussi gestuelles ou physiques. Notre propos se 
démarche de la sociologie compréhensive adoptée ici repose fondamentalement sur «la corrélation du monde et de sa mise en discours par un sujet d'énonciation ${ }^{21}$.

\section{L'apport de la sociologie à l'analyse du discours}

\section{De la linguistique à l'analyse du discours}

Si l'on part d'une définition de l'AD proche de ses origines et du questionnement en termes de formations discursives, on peut en retenir des notions comme celle de «positionnement» et de «conditions d'exercice de la fonction énonciative» qui articulent très directement le discursif au social $^{22}$.

D. Maingueneau définit ainsi les énoncés qui intéressent l'AD:

Il s'agit [...] d'énoncés dont le mode de structuration complexe et relativement stable possède une valeur pour une collectivité, de textes associés à une conviction partagée, qu'ils suscitent et renforcent, en bref de textes qui impliquent un positionnement dans un champ discursif. Le corpus n'y est donc pas considéré pour lui-même, mais en ce qu'il est partie prenante dans une institution reconnue qui «définit pour une aire sociale, économique, géographique ou linguistique donnée les conditions d'exercice de la fonction énonciative ${ }^{23}$.

Dès lors, tant le sujet de cette énonciation, que les conditions qui définissent et contraignent son exercice, sont envisagés en tant qu'ils sont socialement inscrits.

Cette articulation entre les discours et les institutions de production et de diffusion qui les rendent possibles place d'emblée l'AD dans une position décentrée par rapport aux ancrages disciplinaires. Il faut notamment cesser (partiellement) d'être linguiste pour élaborer des hypothèses (construction du corpus, choix des méthodes...), sans quoi la recherche est exposée à une forme de circularité ou de fonctionnement tautologique, où l'on se borne à projeter sur un corpus une méthode préétablie.

concentrera ici sur l'aspect discursif ou linguistique, sachant bien entendu que différents travaux sont allés plus ou moins loin dans la prise en considération de ces différents systèmes de signes.

21. P. Pharo, 1985, «Problèmes empiriques de la sociologie compréhensive», Revue française de sociologie 1, vol. XXVI, p. 127.

22. D. Maingueneau, 1991, p. 17.

23. Ibid., p. 17. La citation de second rang renvoie à M. Foucault, Archéologie du savoir, p. 153. 
Et il faut savoir cesser d'être linguiste au cours de l'analyse elle-même, car la démarche s'alimente à une confrontation constante entre les résultats obtenus et les hypothèses initiales:

Loin d'intervenir les uns à la suite des autres, les divers savoirs sur lesquels s'appuie l'AD doivent être convoqués de façon à se corriger mutuellement, au fur et à mesure que la recherche progresse. Faute de quoi, le résultat de l'analyse risque de n'être qu'une simple projection des hypothèses extra-discursives initiales ${ }^{24}$.

\section{Analyse du discours et démarche ethnographique}

Un concept comme celui d' «ethnolinguistique de l'écrit» ${ }^{25}$ montre comment l'AD a été amenée à reformuler certains de ses concepts fondamentaux, comme celui de formation discursive, en important des problématiques ou des notions issues de la sociologie et/ou de l'ethnographie.

D. Maingueneau, évoquant le «tour ethnolinguistique ${ }^{26}$ pris par l'analyse du discours, signale parmi les déplacements de perspective:

- l'intérêt pour «des groupes restreints, des communautés qui à travers leurs rites langagiers partagent un même territoire»,

- une problématique de l'autorité (notamment dans la filiation de la réflexion de M. Foucault sur la légitimité des énonciateurs),

- mais aussi la notion de «rituel»:

Le texte n'est pas appréhendé comme l'«expression» d'activités muettes placées en amont de lui, mais devient partie prenante d'une pratique qui constitue tout à la fois une dimension du fonctionnement d'un groupe social et son ciment. La mise en scène de l'évènement de parole consolide ou modifie l'espace social, le discours possède un pouvoir d'organisation des hommes (D. Maingueneau, 1992) ${ }^{27}$.

Or dans ce mouvement, l'AD a été conduite d'une part à envisager de nouveaux corpus (discours scientifiques, discours «clos» circulant dans

24. Ibid., p. 25.

25. Voir J.-C. Beacco (dir.), 1992, Langages, 105, « Ethnolin-fuistique de l'écrit », Paris, Larousse.

26. «Le tour ethnolinguistique de l'analyse du discours », Langages 105, 1992, p. 114125. Le tour ethnolinguistique pris par l'analyse de discours correspond à une réaction contre la perspective macrosociologique et la conception représentationniste des textes qui caractérisaient l'AD à la fin des années 1960 .

27. Art. cit., p. 119. 
des communautés restreintes ${ }^{28}$ ) et d'autre part à porter un regard différent sur ses corpus traditionnels, dans lesquels « dire, manière de dire, manière de faire et manière d'être s'enchevêtrent ${ }^{29}$.

Les nouveaux développements de l'AD initiés dès les années 1970 ne se sont d'ailleurs pas limités à la mise en place d'une ethnolinguistique de l'écrit et nous pouvons en suivre les prolongements dans les travaux du groupe Langage et Travail, qui rompent même avec la clôture des corpus : menés dans des environnements où «se présentent pêle-mêle activités matérielles, pratiques sociales non-langagières, manifestations plurisémiotiques (geste, parole, écriture) », ils montrent comment l'infléchissement des problématiques exige de reconsidérer la constitution du corpus elle-même, qui doit être resituée dans le cadre beaucoup plus vaste de la mise en place du dispositif d'enquête:

L'analyse de discours s'est construite, en France, dans un rapport étroit au corpus, dont elle s'est attachée à définir les conditions méthodologiques de validité. Dans la mesure où le corpus renvoyait à des repérages extra-linguistiques préalables, $[\ldots]$ il était généralement constitué à partir de discours préexistants et ne posait guère qu'à la marge des problèmes de choix et de découpage. [...] Situation bien différente de celle qu'on rencontre aujourd'hui dans le domaine du travail, où discours, textes et autres matériaux de l'analyse sont pour une large part à produire par le mouvement de la recherche elle-même. Quand il se propose d'étudier la coopération dans un atelier [...], le chercheur ne peut échapper à la question de la construction des données. [...] La question du corpus se voit replacée dans une problématique plus générale de l'enquête ${ }^{30}$.

\section{Constructivisme et élargissement du contexte}

Une conception constructiviste du sens semble imposer plus impérieusement à l'analyste du discours un élargissement du contexte. Une telle démarche suppose en effet de s'efforcer de restituer les connaissances, savoirs, catégories mobilisés par les sujets parlants pour construire le sens:

Schématiquement il est possible d'opposer des conceptions empiristes de la référence où l'on assimile les référents d'un énoncé aux objets ou évènements du monde extra-linguistique, à des conceptions «constructivistes» où le réfé-

28. Par exemple, les travaux sur l'historiographie de M. de Certeau ou ceux de J.-C. Beacco sur le rhétorique de l'historien.

29. Ibid., p. 123.

30. J. Boutet, B. Gardin, M. Lacoste, «Discours en situation de travail», mars 1995, Langages 117 - «Les analyses du discours en France», p. 13-14. 
rent est compris comme le résultat d'une activité cognitive ou d'un travail des sujets parlants ${ }^{31}$.

Une telle conception rejoint l'évocation par Georges Kleiber du «contextualisme» modéré ou radical, défini par François Récanati: «Selon le contextualisme modéré, les mots ont des significations fixes qui ne se confondent pas avec la valeur sémantique qu'ils endossent contextuellement et qui est engendrée $»^{32}$.

Et dans la perspective du contextualisme radical,

[F. Récanati] invite à voir dans une unité lexicale une clef pour accéder à un ensemble de connaissances encyclopédiques, le contexte linguistique et situationnel se chargeant ensuite d'activer certaines de ces connaissances pour le calcul de l'interprétation désirée. [...] Le co-texte et le contexte [rendent] certains éléments de cette base de données plus saillants que d'autres et plus susceptibles de servir d'ingrédients dans le processus de construction du sens ${ }^{33}$.

En effet, une démarche constructiviste interdit de préjuger du sens en contexte d'une unité lexicale ou d'un énoncé et suppose une exploration des interactions entre énoncé et contexte.

Ainsi, pour emprunter un exemple au travail de Josiane Boutet, une approche strictement linguistique conduirait à écarter a priori des désignations des métiers un terme comme remplaçant ou polyvalent. Pourtant, si l'on met en équivalence, selon des critères formels, les réponses données à la question «quel est votre métier?», on est amené à les inclure dans la catégorie «désignations des métiers», observation qui ne peut être interprétée que grâce à une connaissance approfondie du contexte.

Inversement co-texte et contexte permettent d'éclairer la polysémie de l'expression être qualifié, qui peut renvoyer à des réalités aussi diverses que les dispositifs de formation, les problèmes de l'emploi et de la reconversion, la distribution des postes de travail dans l'entreprise...

De ce point de vue, l'interprétation du sens de l'énoncé ou du texte appelle à un même élargissement du contexte que celle de la signification donnée à un programme télévisuel dans l'optique de Jean-Pierre Esquenazi, qui cite John Dewey pour évoquer le «tout contextuel»:

31. J. Boutet, 1994, 2 éd. 1997, Construire le sens, Berne, Peter Lang, p. 46.

32. F. Récanati, 1997, «La polysémie contre le fixisme», Langue française 113, p. 121, cité par G. Kleiber, 1999, Problèmes de sémantique, la polysémie en questions, Lille, Presses universitaires du Septentrion, p. 43.

33. F. Récanati, 1997, p. 122, cité par G. Kleiber, 1999, p. 44. 
La logique d'une situation est ce qui nait en même temps que l'observation de la situation. Il n'y a pas de logique autre que celle des agents concernés par la situation, qui, donc, lui attribuent un sens. [...] Nous considérerons donc qu'un programme télévisuel doit être inclus dans un «tout contextuel», seul capable de nous faire comprendre la signification qu'il revêt pour les acteurs sociaux engagés, à un titre ou un autre, par ce programme ${ }^{34}$.

Les premiers résultats de notre travail sur les relations entre militaires et médias suggèrent, par exemple, que ces relations imposent de prendre en compte les interactions entre les champs médiatique, militaire et politique, le discours produit par la DICOD ${ }^{35}$ ou les SIRPA, les lectures de la politique de communication de la défense proposées par les professionnels militaires des relations avec les médias, par les journalistes, par la communauté militaire ${ }^{36}$.

En résumé, on peut considérer en se référant à ces évolutions de l'AD qu'elle aborde le discours non seulement en tant qu'il est articulé à des pratiques (du rituel du grand oral - ou de la soutenance ${ }^{37}$ - aux routines administratives, ou à l'exercice quotidien de l'activité professionnelle dans un atelier...) mais qu'elle analyse le discours en tant que pratique.

La notion de formation langagière introduit l'idée qu'il existe des rapports de force entre les pratiques langagières et non pas seulement que le langagier porte trace ou reflète des rapports de force qui lui sont extérieurs [...]. En introduisant la notion de pratiques langagières nous souhaitons aussi dire que le langage est une pratique, et qu'à ce titre il est à concevoir comme partie prenante des autres pratiques sociales, que ce soit des pratiques de production, de transformation ou de reproduction ${ }^{38}$.

34. 1999 Télévision et démocratie. Le politique à la télévision française, 1958-1990, Paris, PUF, p. 16. La situation télévisuelle comprend dès lors: «le contexte médiatique et politique; le discours télévisuel que ce contexte fait naitre; l'ensemble des interprétations dont ce discours est l'objet» (ibid., p. 20).

35. Délégation à l'information et à la communication de la Défense, créée en juillet 1998 en remplacement du SIRPA central. Par ailleurs existent toujours quatre SIRPA d'armées : armée de terre, armée de l'air, marine et gendarmerie.

36. Nous entendrons par discours de la «communauté militaire» celui qui émane des cercles les plus larges de la définition de l'institution proposée plus haut (celui des énonciateurs militaires s'exprimant dans un contexte semi-officiel - articles réputés «libres opinions » de la presse militaire ou articles de la presse militaire non-institutionnelle -, ou dans un contexte officieux - conversations informelles, entretiens anonymés...), par opposition au discours institutionnel au sens strict, qui renvoie pour nous à l'ensemble des positions énonciatives officielles.

37. Voir C. Dardy, D. Ducard, D. Maingueneau, 2002, Un genre universitaire; le rapport de soutenance de thèse, Lille, Presses universitaires du Septentrion.

38. J. Boulet, 1997, p. 61. 
C'est bien dans cette filiation que se situe notre approche, même si notre objet peut apparaitre comme très différent. Comprendre le discours institutionnel nous suggère de prendre en considération l'ensemble des pratiques qui forment «le champ d'intelligibilité où il prend sens ${ }^{39}$.

\section{Ce que l'analyse du discours peut apporter à la sociologie}

\section{Corrélation du monde et de sa mise en discours}

La sociologie compréhensive, comme notamment l'ethnométhodologie, se préoccupe du sens endogène de l'activité sociale, c'est-à-dire de «la façon dont les acteurs rendent visibles pour autrui, notamment par leurs jeux de langage, les activités qu'ils leur destinent ${ }^{40}$ ou pas. À la différence d'une recommandation sociologique traditionnelle du type «ne vous fiez pas à ce qui est dit mais à ce qui est fait»-, l'approche sociologique de type compréhensif travaille le matériau discursif en tant qu'il est une composante du monde social, forte du postulat selon lequel les discours sociaux en tant que conceptions du monde sont une composante de ce monde.

Partant de ce cadre théorique, il importe dans un premier temps de faire le point sur ce qui constitue l'approche du discours en tant que forme langagière par les sociologues. Même si la plupart d'entre eux sont de fait peu diserts sur les modalités de traitement du matériau discursif, on peut mettre au jour que des méthodes et une réelle réflexivité existent. Pour autant, les apports de l'AD à cette démarche sociologique restent à mettre en évidence - et en application -, tout autant d'un point de vue heuristique que méthodologique.

Le discours vu par la sociologie compréhensive: approche thématique, interprétative et objectivante

Quand elle est abordée (dans la partie méthodologique d'un ouvrage ou dans un ouvrage de méthodologie à part entière), l'analyse du corpus de discours du sociologue (entretiens, questions ouvertes ou conversations recueillies in vivo) est le plus souvent traitée selon une approche thématique.

39. M. Senellart, 2001, «Machiavel à l'épreuve de la gouvernementalité», dans G. Sfez et M. Senellart (dir.), L'enjeu Machiavel, Paris, PUF.

40. P. Pharo, 1985, p. 127. 
Ainsi la sociologue de l'art Nathalie Heinich, s'expliquant sur le choix d'une méthode qualitative et sur la démarche d'analyse des entretiensrécits de vie menés avec des lauréats du prix Goncourt:

Déjà, l'analyse thématique esquissée dans les marges des récits d'entretien a permis non pas d'expliquer mais d'expliciter les différents éléments qui interviennent dans une telle expérience, en déployant l'extraordinaire diversité des directions d'analyse contenues dans un seul cas. [...] Il va falloir dégager la cohérence de ces éléments, non en les rabattant sur les classiques «facteurs explicatifs » évoqués à l'instant, mais en dégageant, du corpus lui-même, les grandes lignes sous-jacentes, les problématiques communes qui sous-tendent la logique des thèmes évoqués, et dont l'homogénéité n'est pas ou guère accessible au regard immédiat. L'analyse thématique débouchera ainsi sur une recherche des récurrences, donnant accès à un niveau plus général d'«explication ", par des facteurs non seulement extra-individuels mais aussi, nous allons le voir, largement extra-littéraires ${ }^{41}$.

Sur un autre terrain mais avec une interrogation liminaire analogue portant sur la dialectique entre réussite sociale et identité, notre travail sur les relations entre militaires et journalistes ${ }^{42}$ nous a conduite à mener une série d'entretiens à caractère de récit de vie avec les anciens chefs du SIRPA, l'ensemble constituant finalement un corpus restituant des expériences professionnelles et personnelles à la fois communes, exceptionnelles ${ }^{43}$ et fondamentalement singulières. Le contenu de chaque entretien restait en effet relatif à un contexte politique et militaire, voire diplomatique, circonstancié, chaque énonciateur étant compris à la fois comme un acteur en situation professionnelle distincte et comme un individu aux analyses, aux réactions et à la subjectivité propres.

C'est en tenant compte de ces différents paramètres qu'a d'abord été transcrit, puis lu et relu chaque entretien, pris à la fois dans sa singularité et dans ses relations - souvent explicitement mentionnées par les enquêtés eux-mêmes, fondamentalement réalisées et analysées par nous-même, notamment sous la forme de recoupement - avec l'ensemble du corpus.

41. 1999, L'épreuve de la grandeur - Prix littéraires et reconnaissance, Paris, La Découverte, p. 179.

42. "Quels "professionnels" de la communication et des relations avec les médias à la Défense? - Carrière militaire et communication", Langage et société, op. cit.

43. Ils ont d'abord en commun le fait d'avoir exercé cette fonction identifiée comme une distinction, son caractère exceptionnel constituant lui-même un point commun objectivé par les acteurs. Ils se ressemblent ensuite en raison de leurs trajectoires professionnelles réussies au sein de l'institution, puisque validées par un certain nombre d'attributs militairement et socialement significatifs. 
C'est aussi en faisant en sorte d'objectiver chaque relation d'entretien instaurée depuis chaque sollicitation par courrier jusqu'à la réalisation concrète de l'entretien - et dans quel cadre ou scène symbolique - qu'a été menée l'analyse des entretiens, que l'on peut qualifier de thématique, autour d'entrées signifiantes et de plus en plus détaillées telles que «journalistes», " communication» et «technologies de la communication», « hommes politiques», « vocation militaire», " professionnalisation».

L'évocation de ce travail illustre le fait que la clé de lecture méthodologique du matériau langagier pratiquée par le sociologue est d'ordre thématique, sans pour autant faire strictement référence à l'analyse de contenu thématique telle que l'entendent certains psychosociologues ${ }^{44}$. Existe, en effet, chez un certain nombre de sociologues travaillant sur du matériau discursif, comme chez des ethnométhodologues, le double projet d'interprétation du sens et d'objectivation de l'intervention du chercheur.

Certains de ces travaux cherchent ainsi à se prémunir de toute tentation d'explication du sens, au sens linéaire du terme.

Par ailleurs, bon nombre de sociologues se sont penchés sur le caractère potentiellement artéfactuel ou biaisant de la nature même du dispositif de recherche par entretien. À la différence du travail ethnologique ou anthropologique classique fondé sur l'observation, ce dispositif méthodologique est une sollicitation qui vise à susciter, voire à provoquer une parole et un discours de la part d'acteurs sociaux.

L'observation in situ n'est pour autant ni impossible, ni interdite au sociologue, même si elle demeure plus délicate pour celui qui travaille sur les institutions. Tout un courant de la sociologie qualitative appelle l'immersion du chercheur dans son terrain d'étude, à côté et même aux côtés de ceux dont il observe les interactions en même temps qu'il y est confronté et éventuellement intégré ${ }^{45}$. Comme l'écrit de manière plus générale H. S. Becker, la justesse de «l'imputation de sens et de motifs aux acteurs sociaux» est largement conditionnée par la possibilité de «rassembler des données sur le sens que les gens donnent aux choses» ${ }^{46}$.

Ainsi avons-nous largement fondé un travail portant sur ce qu'il est convenu d'appeler la communication gouvernementale sur une insertion

44. Pour une définition canonique de la démarche, voir en particulier L. Bardin, 1977, L'analyse de contenu, Paris, PUF.

45. Voir en particulier l'ouvrage de S. Beaud et F. Weber, 1998, Guide de l'enquête de terrain, Paris, La Découverte et pour une application, de S. Beaud et M. Pialoux, 2001, Retour sur la condition ouvrière - Enquête aux usines Peugeot de Sochaux-Montbéliard, Paris, Fayard.

46. 2002, p. 41. 
au sein de l'organe politico-administratif ${ }^{47}$ dont l'existence témoignait de la reconnaissance, de l'institutionnalisation et de la relative professionnalisation du phénomène étudié et par la suite replacé dans un cadre interprétatif plus général de «dispositif d'information d'État» ${ }^{48}$.

À côté, mais de manière cohérente avec ce «tour ${ }^{49}$ ethnologique ou «ethnographique ${ }^{50}$ de la sociologie, se sont développées de nombreuses réflexions sur la méthodologie de l'entretien en tant que discours suscité. L'objectivation de la relation entre enquêteur-chercheur et enquêté, au cours de l'entretien, a ainsi été largement traitée dans plusieurs articles ${ }^{51}$, notamment à la suite du chapitre méthodologique et réflexif de La misère $d u$ monde $^{52}$. Dans tous ces travaux, le travail de réflexivité consiste à «rapporter l'enquête à ses conditions sociales de possibilité. Parmi ces conditions, les caractéristiques objectives de l'enquêteur, telles qu'elles sont perçues par les enquêtés, sont importantes: non pas pour ellesmêmes, mais dans l'analyse des multiples interactions d'enquête ${ }^{53}$.

L'entretien est par suite compris non comme une simple technique mais bien comme relation sociale. L'objectivation de ce principe, au travers de ses manifestations langagières, aide à l'interprétation du discours produit au cours de l'interaction d'entretien. Le discours obtenu et recueilli, sous la forme de réponses ainsi que de questions et de relances, est ainsi d'une certaine manière rapporté à ses conditions d'énonciation.

Il apparait, pour ces différentes raisons, que le discours produit par entretien n'est ni observé complètement naïvement, ni surtout selon les catégories de sincérité/mensonge ou, pire encore, de prétendue transparence de l'enquêté et de son discours.

47. Par une activité professionnelle, à temps partiel, de mars 1995 à septembre 1997, au sein du Service d'Information du Gouvernement, rattaché au Secrétariat général du Gouvernement et placé sous l'autorité du Premier ministre pour la coordination de la communication gouvernementale.

48. C. Ollivier-Yaniv, 2000, L'État communiquant, Paris, PUF.

49. Pour paraphraser le titre de l'article de D. Maingueneau portant sur «le tour ethnolinguistique de l'analyse du discours».

50. Voir notamment la postface de S. Beaud et F. Weber: «Pour une ethnographie sociologique» (1998, p. 293-314).

51. Voir M. Pinçon et M. Pinçon-Charlot, mars 1991, «Pratiques d'enquête dans l'aristocratie et la grande bourgeoisie: distance sociale et conditions spécifiques de l'entretien semi-directif», Genèses 3, p. 120-133 ; H. Chamboredon, F. Pavis, M. Surdez, L. Willemez, juin 1994, «S'imposer aux imposants», Genèses 16, p. 114-132; J.-B. Legavre, $3^{\mathrm{e}}$ trimestre 1995, «La neutralité dans l'entretien», Politix 35.

52. P. Bourdieu, 1993, «Comprendre», Paris, Le Seuil, p. 1389-1447.

53. S. Beaud et F. Weber, p. 297. 
Pour reprendre l'exemple du travail sur les relations entre l'armée et les médias, il ne saurait être question d'analyser un corpus d'entretiens avec des officiers supérieurs de l'armée de terre sans prendre en considération le fait que l'enquêteur était un enseignant-chercheur, potentiellement perçu comme représentant de l'institution universitaire et comme analyste des phénomènes d'information et de communication: ce premier paramètre a certainement motivé un certain nombre de propos spontanément ${ }^{54}$ très analytiques, voire conceptuels, sur la communication en général, sur ses technologies et sur la question de sa professionnalisation. Pas question non plus d'oublier que l'enquêteur était aussi... une enquêtrice - figure relativement étrangère au milieu militaire, à destination de qui on met plus particulièrement en avant les caractéristiques purement militaires et opérationnelles de la vocation d'officier, alors même que le principe d'une carrière réussie repose sur de longues périodes «de moquette ${ }^{55}$.

\section{Étude sociologique du discours et analyse du discours}

Pour autant, l'AD permet d'alimenter une approche du discours qui soit encore moins «naïve» et qui en fasse non pas l'enveloppe d'un sens mais le lieu même de son inscription, de sorte qu'il est impossible de séparer l'énoncé de l'énonciation ${ }^{56}$.

On sait que l'AD s'est définie en grande partie contre l'analyse de contenu; le simple terme de «contenu» est d'ailleurs antinomique avec les positions fondatrices de l'AD. Régine Robin a marqué, dès 1973, les limites très étroites dans lesquelles les analyses thématiques enfermaient l'appréhension du discours ${ }^{57}$.

Non que l'analyse thématique soit impossible, mais elle doit expliciter

54. C'est-à-dire sans sollicitation explicite de l'enquêteur.

55. Pour reprendre une expression couramment utilisée en milieu militaire (et repérée par la dite enquêtrice!), toujours pénalisante et stigmatisante, visant à signifier les périodes en état-major, en cabinet et en dehors de tout terrain d'opération.

56. Les remarques qui suivent ne prétendent nullement à l'exhaustivité. Nous mentionnons ici les principales pistes qui nous ont semblé intéressantes pour la poursuite de notre travail.

57. «Toutes ces études, au-delà de leur rigueur, de leurs mérites qui sont immenses, reposent malgré tout sur le postulat de l'immédiateté du sens, et son univocité. [...] Dans tous les cas, il s'agit, selon l'expression de M. Pêcheux, "d'accéder au sens en traversant la structure linguistique du texte". De plus, ces études négligent le niveau discursif en tant que tel, comme si les idéologies ne se donnaient pas aussi en tant que système de représentations dans des discours et comme si l'ordre du discours, sa structure ne comportait pas 
et élucider à mesure ses modalités ${ }^{58}$. De ce point de vue, les concepts et les méthodes élaborés par l'AD pourraient être mobilisés avec profit pour le travail d'analyse des entretiens.

L'analyse de contenu recourt, par exemple, à des regroupements fondés sur la synonymie (utilisant la substitution d'un énoncé à un autre comme un outil) alors que l'AD considère ces substitutions dans un texte comme des paraphrases, comme un fait discursif dont il faut rendre compte. Elle procède, par exemple, par analyse des reformulations, des «paradigmes désignationnels ${ }^{59}$, c'est-à-dire prend acte de la dispersion lexicale observée et tente de lui donner sens au lieu de la réduire à l'unité en fonction d'un sens préétabli.

L'analyse de ces reformulations, de même que de l'ensemble des «non-coïncidences du dire ${ }^{60}$ (phénomènes d'autocorrection, de rectification ou commentaires métadiscursifs), peut trouver dans l'AD des outils qui autorisent une approche élaborée des entretiens :

On ne faisait pas la communication du ministre - au sens des messages - mais on fournissait la logistique. J'avais un service, qui s'appelait le COPID, il fournissait donc la logistique. C'est-à-dire qu'il préparait les dossiers de presse, etc, mais il ne faisait ni la stratégie de communication, ni les messages. Il a fait en sorte que tout ce qui était fondateur de la Défense, c'est-à-dire que toute la stratégie de dissuasion ne soit pas mise à la casserole ${ }^{61}$.

Enfin, l'AD, surtout si elle est menée dans la perspective que D. Maingueneau qualifie d' "analytique ${ }^{62}$, peut permettre une réflexion non pas seulement sur le discours étudié, mais sur son envers : les silences de l'inénonçable.

L'approche «analytique réaliste» recherche en effet les «points d'inconsistance discursive» :

d'implications idéologiques» (R. Robin, 1973, Histoire et linguistique, Paris, A. Colin, p. 62. La citation de second rang est empruntée à M. Pêcheux, 1969, L'analyse automatique $d u$ discours, Paris, Dunod, p. 4).

58. Nous pouvons en citer pour exemple la réflexion proposée sur ce point par N. Heinich, que nous avons citée plus haut.

59. M.-F. Mortureux, 1993, «Paradigmes désignationnels», SEMEN 8, Annales Littéraires de l'Université de Besançon, Paris, Les Belles Lettres, p. 121-142.

60. J. Authier-Revuz, 1995, Ces mots qui ne vont pas de soi. Boucles réflexives et noncoïncidences du dire, Paris, Larousse.

61. Ces exemples sont empruntés au corpus d'entretiens recueillis par C. Ollivier-Yaniv auprès des anciens chefs du SIRPA.

62. Sur la distinction entre approche analytique (réaliste $v s$ représentative) et approche intégrative, voir D. Maingueneau, 1991, p. 26-27. 
Passent alors au premier plan les phénomènes d'hétérogénéité énonciative (marques de mise à distance, reformulations, autocorrections, mélange de sources d'énonciation...) ou les discontinuités presque imperceptibles (les «oublis» d'une énumération, les sauts dans une argumentation, les glissements de signifiants... $)^{63}$.

On peut considérer en effet que le sens à restituer réside aussi (surtout?) dans le non-dit sous plusieurs de ses formes:

- les «omissions», qui peuvent être identifiées et/ou mesurées par simple confrontation lexicale (comparaison du traitement d'un évènement dans plusieurs journaux, des campagnes de plusieurs candidats...),

- le sous-entendu, qui selon Oswald Ducrot, est la réponse à la question: «Pourquoi L. a-t-il dit cela...? ${ }^{64}$ (ce qui renvoie à peu près à ce qui a été dit plus haut de l'élargissement du contexte),

- les références aveugles de l'énonciation: J. Boutet considère à la suite de Pierre Fiala ${ }^{65}$ que «l'hétérologie», «constitutive de toute interaction », peut prendre trois formes: le dialogisme direct, l'intertextualité (du mot rapporté à l'allusion) et les formes où le discours d'autrui n'est pas reconnaissable et fonctionne comme «référence aveugle de l'énonciation"».

Ainsi le discours des ouvriers, étudié par J. Boutet, apparait-il comme une réponse à la voix patronale absente.

Si toute parole est une «contre-parole», la construction du sens exige de restituer l'ensemble des voix citées ou évoquées mais aussi plus généralement l'ensemble des «voix concurrentes ou convergentes qui circulent ${ }^{66}$.

Une étude de la communication de la défense pendant l'année 2001 peut-elle, par exemple, être menée sans envisager les voix «concurrentes » qui évoquent au même moment la torture en Algérie, thème généralement contourné par le discours officiel militaire?

63. D. Maingueneau, 1991, p. 27. Ici encore, nous emprunterons une illustration aux entretiens déjà cités: «Chaque ministre, surtout chaque équipe ministérielle - vous savez, c'est le problème des entourages - ils ont envie, parce que le SIRPA a les moyens, quand même, et plus l'ECPA [Établissement cinématographique et photographique des armées], ils ont envie que cet outil-là soit utilisé pour... j'allais dire la promotion du ministre. C'est humain».

« Pour lui succéder, on a pris un contrôleur général pour la première fois - j'allais dire à ma grande surprise».

64. O. Ducrot, 1984, Le dire et le dit, Paris, Minuit, p. 13-46.

65. P. Fiala, 1986, «Polyphonie et stabilisation de la référence: l'altérité dans le texte politique», Travaux du Centre de recherches sémiologiques, 50, p. 15-46.

66. J. Boutet, 1997, p. 170-180., 
«L’inénonçable» apparait en effet comme une dimension essentielle de la définition de l'énonciation légitime pour un sujet (ou un groupe) donné dans une situation donnée:

L'objet de l'AD n'est pas tant la formation discursive elle-même que sa frontière constitutive. [...] L'énonciation ne se développe pas sur la ligne d'une intention fermée sur son propre vouloir, elle est de part en part traversée par la menace du glissement dans ce qu'il ne faut pas dire, dans ce qu'il ne faut surtout pas dire, dont la présence à la fois envahissante et invisible double constamment l'énonciation légitime dès qu'elle surgit ${ }^{67}$.

Une telle conception n'est-elle pas de nature à intéresser ceux des sociologues qui pensent avec Georg Simmel que «si la socialisation humaine est déterminée par la capacité de parler, elle est modelée par la capacité de se taire ${ }^{68}$ ?

\section{Vers une anthropologie des discours institutionnels}

La réflexion présentée ici s'efforce de rapprocher des corpus, des méthodes, et surtout des concepts. Ce travail représente une étape et la démarche demande encore à être précisée, en particulier au travers de différentes formes de mise en œuvre ${ }^{69}$.

Pour ce qui concerne le discours des militaires et la question des relations entre armée et médias, nous comptons aborder la question des relations entre militaires et journalistes, cette fois du point de vue des journalistes. Dans ce cas, la méthode d'analyse du discours institutionnel aura pour principal infléchissement d'élargir la définition de l'institution selon la conception définie en introduction et de se porter du cercle le plus étroit de l'institution au cercle le plus large: il existe en effet un discours de type institutionnel produit par et autour de la profession de journaliste. Nous ne prendrons pour exemples que la récente publication par Le Monde d'un fascicule intitulé Le style du monde et qui constitue une forme de charte de l'écriture pour la rédaction, mais aussi et surtout la littérature pédagogique produite par les écoles de journalisme qui font référence ${ }^{70}$. L'approche à partir d'entretiens reste à préciser par la définition de la popula-

67. D. Maingueneau, 1991, p. 20-21.

68. G. Simmel, 1996, Secret et sociétés secrètes, Paris, Circé, p. 111.

69. Et notamment par le biais d'un séminaire de recherche mené conjointement par les auteures à l'École militaire spéciale de Saint-Cyr.

70. C. Oger a par exemple travaillé sur les fascicules publiés par le CFPJ en s'exerçant 
tion à enquêter - l'élite des journalistes de défense, très peu nombreux, la population plus vaste mais plus mouvante des accrédités auprès de la DICOD, et/ou encore les correspondants de guerre -, ainsi que par les modalités d'analyse et de traitement du corpus d'entretiens.

Enfin et de manière plus générale, cette démarche nous semble valide pour aborder d'autres formes de discours à caractère institutionnel. Avec une telle perspective croisée, c'est une anthropologie des discours institutionnels qu'il nous semble possible d'envisager, et ceci avec un double projet: éclairer un certain nombre de discours institutionnels en intégrant leurs conditions de production par la sollicitation des acteurs sociaux impliqués; dénouer ou pour le moins identifier les arcanes de tout phénomène énonciatif, qu'il soit porté par un collectif ou par un individu représentatif de ce «groupement social légitimé» ${ }^{71}$.

à la construction d'un modèle discursif sous-jacent et à sa confrontation systématique avec le modèle discursif dégagé des textes institutionnels et des méthodes militaires. Ce travail n'est pas encore publié.

71. M. Douglas, 1999, p. 17. 